\title{
فعالية استخدام المواد التعليمية على أساس الدراسة التقابلية في تعليم مهارة الكتابة بقعيم تعليه اللغة العربية جامعة مولانا مالك إبراهيم الإسلامية الحكومية مالانج \\ إعداد: ديه دين أمينة \\ كلية الدراسة الإسلامية بجامعة مالانج الإسلامية دائة \\ Email:diahdina@unisma.ac.id
}

Abstract

Most of university students use Indonesian language structure in Arabic language writing. So, those sentences become false. The university student just obey to the lesson book that used in learning process as the common, whereas that lesson book still doesn't pay attention of the differences and the similar from the structure of the main languages and the language that they are studied. Contrastive analysis is an activity to find both of the differences and similar of two languages and also as the solution of the languages' problem. So, the researcher want to know the effectivity of the lesson book of writing skill by using contrastive analysis theory in Arabic teaching department of State Islamic University of Maulana Malik Ibrahim Malang.

Based on that thinking, this research's purposes are to know about The effectivity of lesson book on writing skill by using Contrastive Analysis. This research uses quantitative approach. And this research's kind of method is experiment model. For the analysis of this research's data, are used inferential statistic analysis by using $t$ test.

The results of this research is The researcher use test of two chapters using from the teaching material with intact group comparison method, that is by dividing the class become two groups, control group and experiment group. In the test of the first chapter, the score is 26,6 (t-arithmetic) > 1,27 ( $t$-table), it means that this teaching material is effective. In the test of the second chapter, the researcher gets the score 16,5 (t-arithmetic) $>1,27$ ( $t$-table), it means that this this teaching material is effective.

$$
\text { الكلمات الأساسية: مهارة الكتابة، الدراسة التقابلية. }
$$

إن الكتابة هي إحدى وسائل الاتصال للإنسان سوى الكلام، وأراد الإنسان أن يخبر غيره

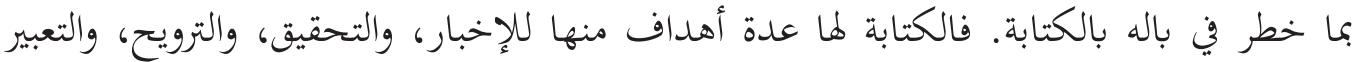


(Henry Guntur Tarigan)

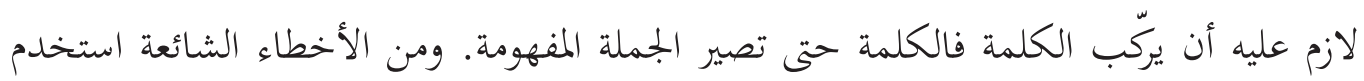

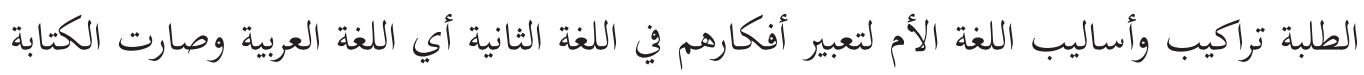

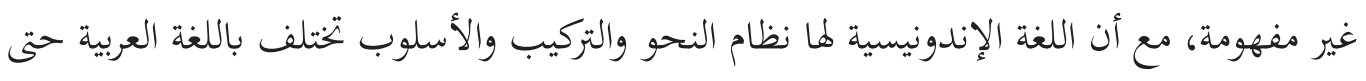
تكون هذه الفروق سبب أخطائهم في الكتابة.

فتعوّد الطلبة بمفهوم اللغة الأصلية في جميع العناصر اللغوية مثل الأصوات والنحو والصرف

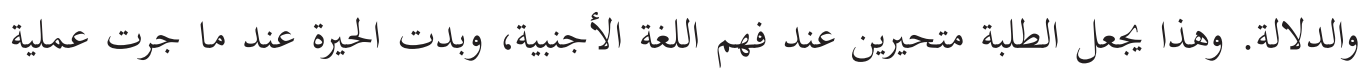

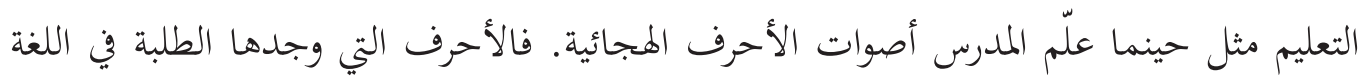

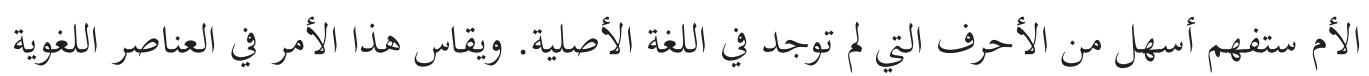

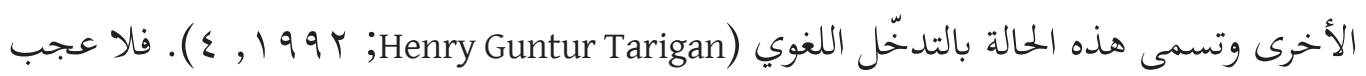
إن وجدت الصعوبات عند الطلبة والمدرس في عملية تعليم اللغة الأجنبية.

الدراسة التقابلية هي أنشطة التقابلية بين الجملتين أو أكثر لمعرفة الفرق بينهم. أي بهذا



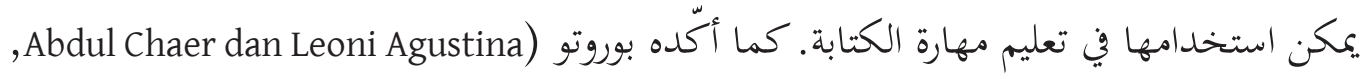

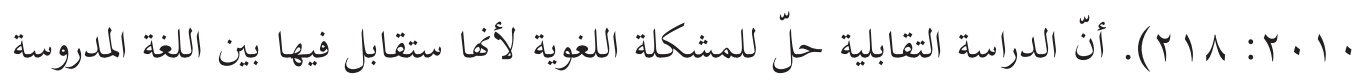

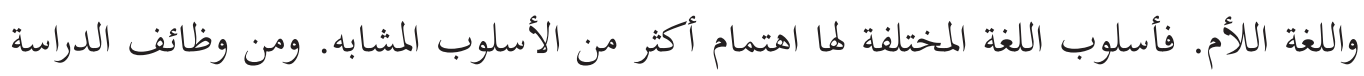

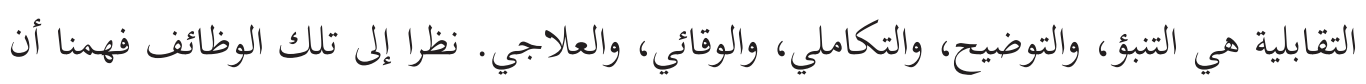

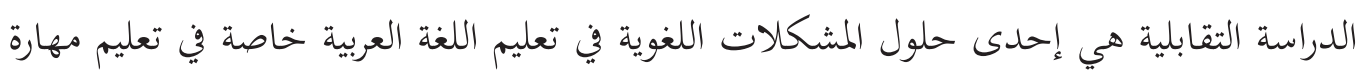
الكتابة.

فعملية التعليم أيا كان نوعها أو نمطها أو مادمّا ومحتواها تعتمد كبيرا على الكتاب التعليمي،

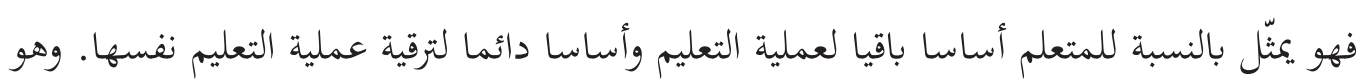

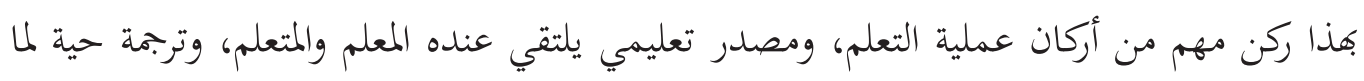



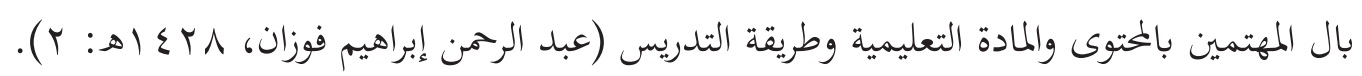


وتزداد أهمية المواد التعليمية لتعليم الكتابة المبني على أسس لغوية ونفسية وثقافية. ويواجه المعلم دائما بالحاجة إلى إعداد كتب دراسية ومواد تعليمية متطورة تفي بحاجة نوعيات خاصة من

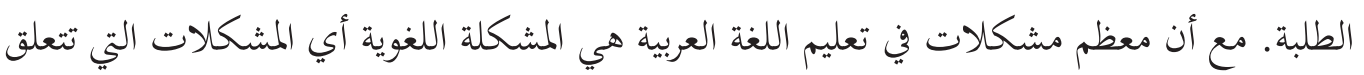

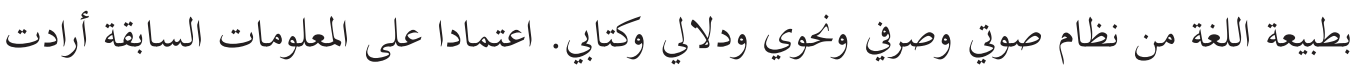

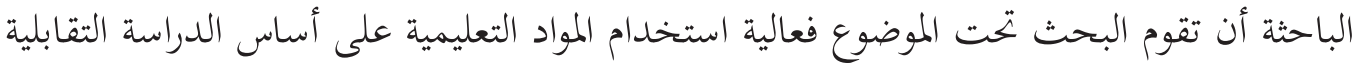
في تعليم مهارة الكتابة.

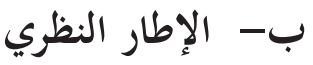

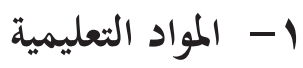

إن المواد التعليمية على درجة كبيرة من الأهمية، وتأتي في المرتبة الأولى قبل أدوات الأجهزة،

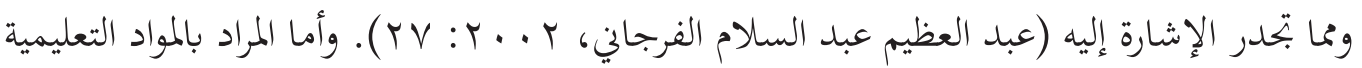

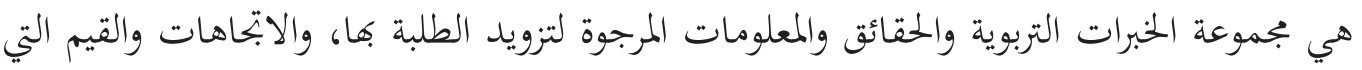

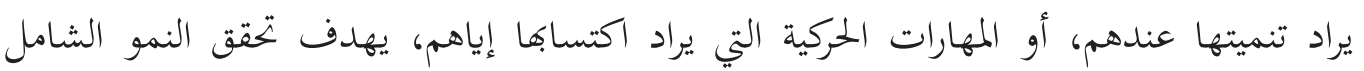

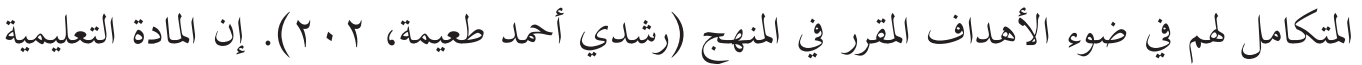

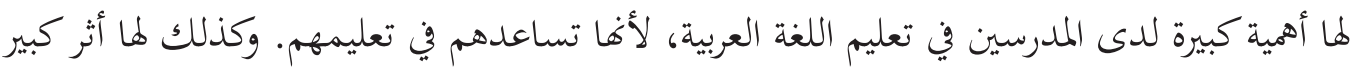

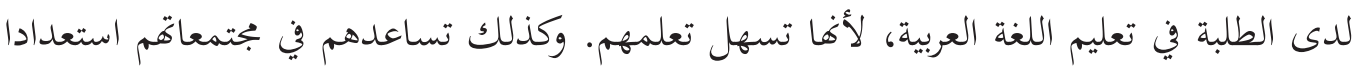
للتعبير عن أفعالمم المحدودة وتعلم المعلومات وتقديمها باللغة العربية، دون تفكير في لعيم اللغة نفسها.

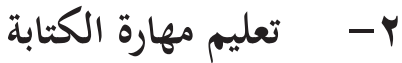

فمفهوم مهارة الكتابة في بعض البرامج ليقتصر على النسخ copying أو التهجئة spelling.

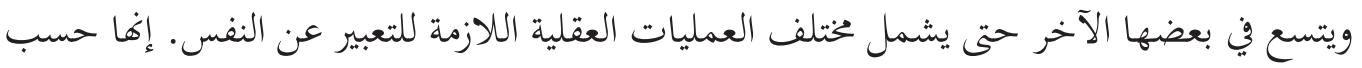

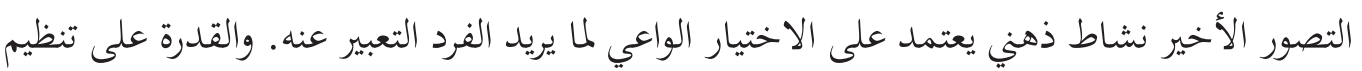

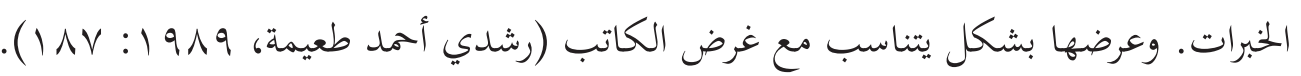
الكتابة هي عملية معقدة، في ذاتما كفاءة أو قدرة على تصوّر الأفكار وتصويرها في حروف 
وكلمات وتراكيب صحيحة، وفي أساليب متنوعة مدى العمق والطلاقة مع عرض تلك الأفكار في

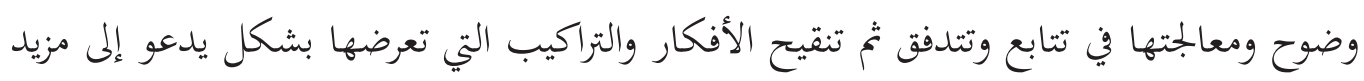
من الضبط والتفكير (إبراهيم علي ربابعة، 0).

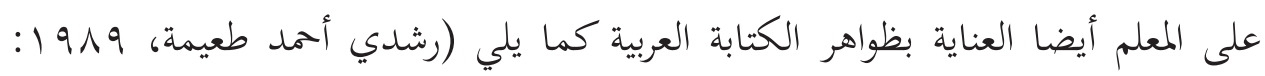

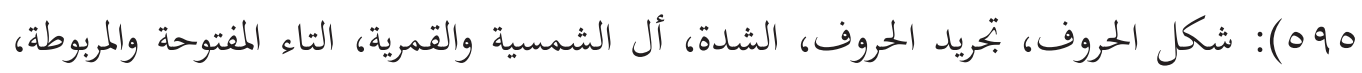

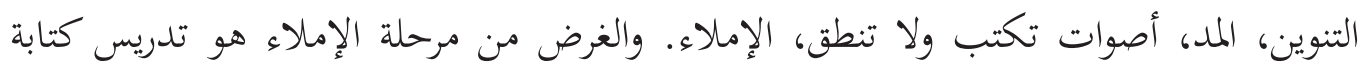

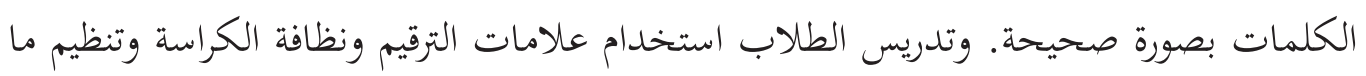
يكتب.

بعد أن يتعلم الطلاب كتابة الحروف والنسخ والإملاء، يمكن أن تبدأ الكتابة المقيّدة التي تسمى أيضا الكتابة الموجهة. وهي مرحلة تسبق التعبير الحر. و وتأتي الكتابة الحرة في المرحلة الأخيرة

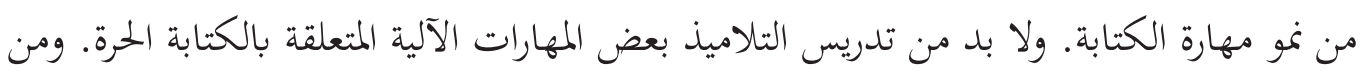

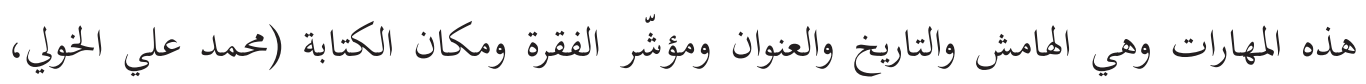
.(1) : : 1914

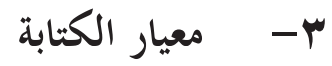

يقوس فتحي علي يونس ومحمد عبد الرؤوف المستويات في تقدير درجات التعبير كما يلي

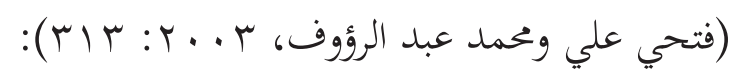

\begin{tabular}{|c|c|c|}
\hline البيان & الدرجات & المستوى \\
\hline • • الجأخمل بسيطة & $7-0$ & مقبول \\
\hline • • • التراكيب اللغوية جيدة & $\Lambda-V$ & جيد \\
\hline
\end{tabular}




\begin{tabular}{|c|c|c|}
\hline • • • • • قجفردات ليست قليلة & 9 & جيد جدا \\
\hline 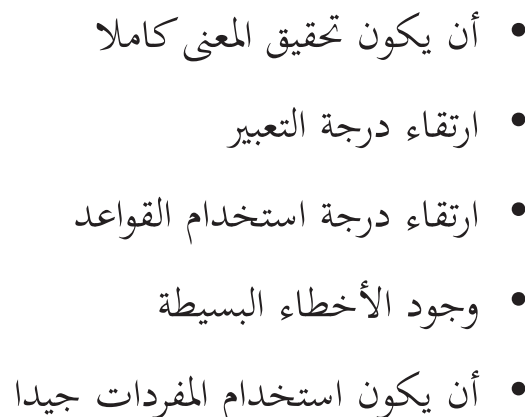 & 1. & محتاز \\
\hline
\end{tabular}

جدول 1: - معيار مهارة الكتابة

\section{ع - ع - الدراسة الثقابلية}

الدراسة التقابلية لغة مأخوذة من كلمة التقابل أي (contrastive) في اللغة الإنجليزية بمعنى

$$
\text { أ) أختلاف أي متعارض. أما عند الاصطلاح قد قدّم بعض العلماء آراءهم منها: }
$$

إجراء عملي للمقارنة بين أنظمة لغتين أو أكثر لحصر أوجه التشابه وأوجه الاختلاف بينهما. ويعتمد ذلك على تحليل لكل من النظامين موضع المقارنة يقوم على أساس من المنهج الوصفي لا التارخي

$$
\text { ب) (بوس دنيل فريرا }
$$

الدراسة التقابلية هي أنشطة التقابل بين اللغة السليقة واللغة الثانية في تركيبهما وقواعد اللغة

المتفق. (Jos Daniel Parera, 1997: 44)

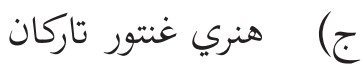

الدراسة التقابلية هي محاولة للتقابل بين تركيب اللغة السليقة واللغة الثانية لمعرفة الاختلافات

(Henry Guntur Tarigan, 1992:4 ).بينهم 
اعتمادا على ما قدّمه اللغويون أن الدراسة الثقابلية هي أنشطة التقابل بين اللغتين أو أكثر لمعرفة المتشاهات والاختلافات بين ذات اللغات. فبروز الدراسة التقابلية يتعلق بعلم اللغة البنائية.

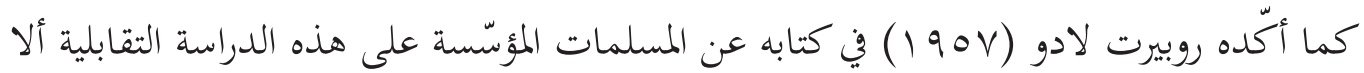
وهي: أن المواد الدراسية التي أكثر فعالية هي المواد المؤسّسة على وصف اللغة المدروسة مع المقارنة الدقيقة بوصف اللغة الأم.

ب) أن أجزاء اللغة المدروسة المتشاهة بللغة الأم تكون سهلة عند الطلبة وكذا بالعكس. ج) سيفهم معلمو اللغة الثانية فهما جيدا عن المشكلات الموجودة عند عملية تعليم اللغة الثانية. د) أغلب الطلبة يتحولون اللغة المدروسة أي اللغة الثانية إلى اللغة الأم أي اللغة الأولى إنتاجية أو استقبالية.

فبرزت الدراسة التقابلية كعلم اللغة التطبيقي الذي له نظرية وتطبيق علمية. وبروز الدراسة التقابلية في مجال تعليم اللغة العربية محتاج إلى اهتمام كبير نظرا إلى ثنائي اللغة الذي لارد له.

\section{ج- منهجية البحث

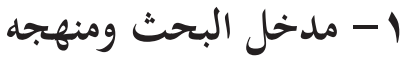

فالمدخل المناسب لهذا البحث هو المدخل الكمي. يهدف هذا المدخل لمعرفة علاقة بين

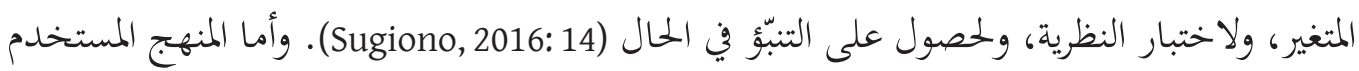
في هذا البحث هو المنهج التجريبي (experiment). يهدف المنهج التجريبي هو لاختبار البيانات التنبئية من البيانات الحاصلة من التجربة عبر المرافقة والملاحظة جميع المتغير المتعلقة.

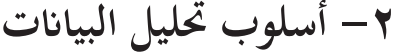

والمراد بتحليل البيانات هي العملية التي تنظم أو تكون بواسطها الملاحظات النتيجة عن

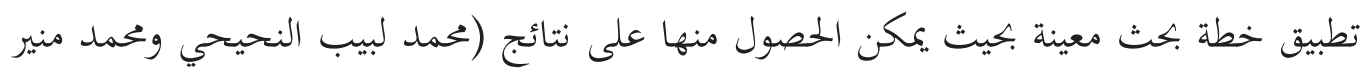




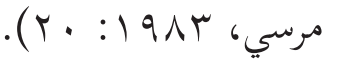

ولتحليل البيانات الكمية تستعمل الباحثة تحليل إحصائي الاستدلالي (Analysis Statistic) (Inferensial Population) والتي سبقت الإشارة إليها، أي اختبار جماعة صغيرة تمثل المجتمع الكبير (Sampling) (or Universe

وإذا أردنا الحصول على المقارنة بين العينتين الفارقتين فنستعمل اختبار T للمجموعة المستقلة Anas Sudijono كأن الاختبار T هو الاختبار الفرض (Independent Groups T-Test)

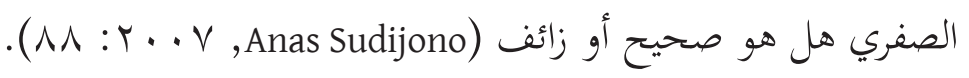

وفي هذه الحالة تستخدم الباحثة أسلوب تحليل البيانات عن نتيجة الطلبة التي تتجلى من فروق المقياس المعدل لنتيجة الطلبة حتى تختبر فروض البحث تؤخذ مستوى الفروق ( • ) في المائة، ولتحليل مستوى فروق نتيجة التعليم للاختبارين تقوم الباحثة على الخطوات التالية:

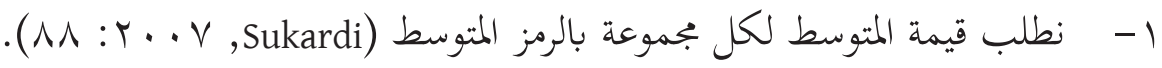
r- وبعد ذلك نطلب قيمة الانحراف المعياري بين قيمة المتوسط في المجموعة الضابطة وقيمة المتوسط في المجموعة التجريبية.

r- نطلب مستوى الفروق بين الجمموعة التجربية والمجموعة الضابطة (Suharsimi Arikunto:

$$
\begin{aligned}
& \text { • } 19 \text { 1 | (9 1). وتمتحن بعد ذلك نتيجة مستوى الفروق بقيمة (T-Test). } \\
& t=\frac{M d}{\sqrt{\frac{\sum X^{t}}{N(N-1)}}}
\end{aligned}
$$

عرضت الباحثة البيانات المتعلقة بفعالية المواد التعليمية على أساس الدراسة التقابلية في تعليم مهارة الكتابة لدى طلبة المرحلة الرابعة من قسم تعليم اللغة العربية بجامعة مولانا مالك إبراهيم الإسلامية الحكومية مالانج. وهذه التجربة تقام في موضوعين من المواد التعليمية. واستخدمت 
الباحثة أسلوب intac group comparison وهو تقسيم فصل واحد إلى بجموعين، مجموع الضابط

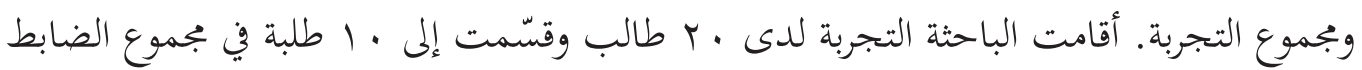
و • ا طلبة في جمموع التجربة. فحصلت الباحثة على البيانات التالية:

أقامت الباحثة التجربة الميدانية الأولى واستخدمت الباحثة الدرس الثالث بموضوع وصف

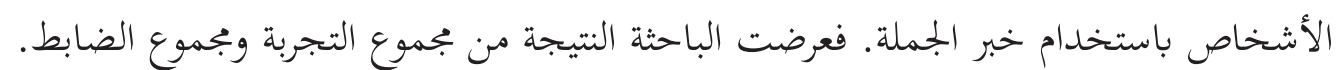
وجدت الباحثة أن نتيجة مجموع التجربة دلت على ع طلبة أو • ع ٪ حصلوا على تقدير

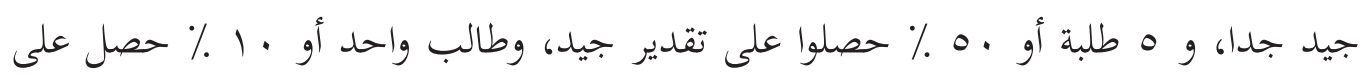
تقدير مقبول.

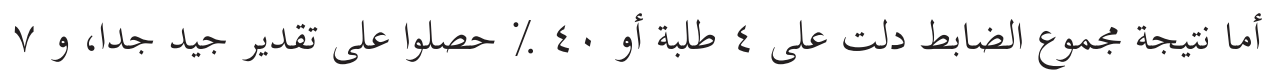

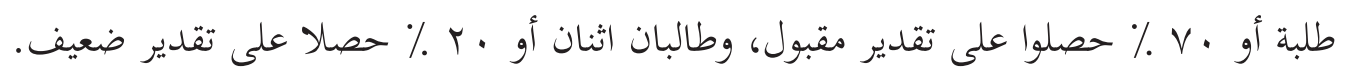
وبعد عرض الباحثة نتيجة مجموع الضابط ومجموع التجربة. أقامت الباحثة المقارنة بين نتيجة

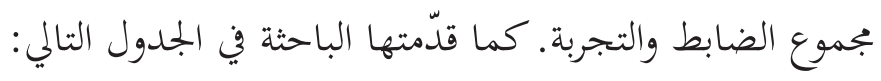

\begin{tabular}{|c|c|c|c|c|}
\hline$d^{\wedge} 2$ & D & $\mathrm{Y}$ & $\mathrm{X}$ & الرقم \\
\hline OVT & $r \varepsilon$ & VI & $1 \ldots$ & 1 \\
\hline$\varepsilon \ldots$ & $r$. & VY & $9 r$ & r \\
\hline rot & 17 & VY & $\Lambda \wedge$ & $r$ \\
\hline rTo & 10 & VY & $\Lambda V$ & $\varepsilon$ \\
\hline Y0T & 17 & 71 & $\Lambda \varepsilon$ & 0 \\
\hline$\varepsilon \ldots$ & $r \cdot$ & $7 \varepsilon$ & $\Lambda \varepsilon$ & 7 \\
\hline$\varepsilon \ldots$ & $r$. & $7 \varepsilon$ & $\wedge \varepsilon$ & V \\
\hline rot & 17 & 7. & $V 7$ & $\Lambda$ \\
\hline $1 \cdot r \leq$ & rr & $\varepsilon \varepsilon$ & $V_{7}$ & 9 \\
\hline $19_{97}$ & ry & ru & Vr & 1. \\
\hline
\end{tabular}




\begin{tabular}{|c|c|c|c|c|}
\hline 0.19 & $r 10$ & Tr人 & $\Lambda \varepsilon r$ & المجموع \\
\hline 0.169 & $Y 160$ & $7 r_{6} 1$ & N & المتوسط \\
\hline
\end{tabular}

جدول r : مقارنة نتيجة بجموع الضابط والتجربة من الموضوع الأول

$$
\text { البيانات: }
$$

X

Y

\section{D : التفاوت بين نتيجة مجموع الضابط وبجموع التجربة}

بناء على العرض السابق وجدت الباحثة التفاوت بين نتيجة بجموع الضابط ونتيجة مجموع


وحصلت الباحثة التفاوت بينها على 0، ا ب . ولتحليل تلك النتائج استخدمت الباحثة معادلة التاء

$$
t=\frac{M d}{\sqrt{\frac{\sum x^{2} d}{N(N-1)}}}
$$

: : Md

: : $\quad \sum x^{2} d$

$$
\begin{aligned}
& \text { : ملدد أفراد العينة N N } \\
& t=\frac{62.8}{\sqrt{\frac{508.9}{10(10-1)}}}
\end{aligned}
$$

$$
t=\frac{62.8}{\sqrt{\frac{508 ، 9}{10(9)}}}
$$




$$
\begin{array}{r}
t=\frac{62.8}{\sqrt{\frac{508.9}{90}}} \\
t=\frac{62.8}{\sqrt{5.65}} \\
26.6=\frac{62.8}{2.36}
\end{array}
$$

لما كانت فرضية البحث التي قدمتها الباحثة هي أن المواد التعليمية التي استخدمتها الباحثة لدى طلبة المرحلة الرابعة من قسم تعليم اللغة العربية بجامعة مولانا مالك إبراهيم الإسلامية الحكومية مالانج فعالة في تعليم كتابة الجملة العربية فأصبح المعيار لتصديق هذا الفرض كما يلي: إذا كانت درجة تاء الحساب أكبر من درجة تاء الجدول (t-table) فالفرض مقبول، أي أن المواد التعليمية المستخدمة فعالة في تعليم كتابة الجملة العربية. ب) إذا كانت درجة تاء الحساب أصغر من درجة تاء الجدول (t-table) فالفرض مرفوض، أي أن المواد التعليمية المستخدمة فعالة في تعليم كتابة الجملة العربية. ج) جبل أن تختبر الباحثة فرضية البحث بذلك المعيار، عرضت الباحثة البيانات الآتية:

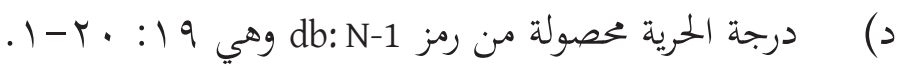

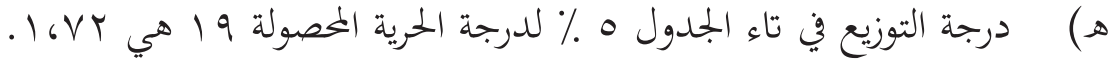

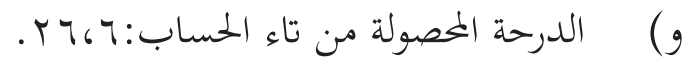

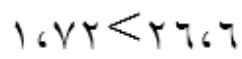

حصلت الباحثة من العرض السابق أن درجة تاء الحساب (7، Y Y) أكبر من تاء الجلدول على مستوى التصديق ه \% ( IVY ). بمعنى أن الفرض مقبول أي أن المواد التعليمية المستخدمة فعالة في تعليم كتابة الجملة العربية.

قامت الباحثة التجربة الميدانية الثانية، واستخدمت الباحثة الدرس السابع بموضوع مشكلة وأسباها باستخدام المفعولين. فعرضت الباحثة النتيجة من مجموع التجربة ومجموع الضابط كما يلي: 
إن نتيجة بجموع التجربة دلت على ه طلبة أو ه . ٪ حصلوا على تقدير جيد جدا، و ع طلبة أو ، ع ٪ حصلوا على تقدير جيد، وطالب واحد أو · 1 ٪ حصل على تقدير مقبول. أما نتيجة مجموع الضابط دلت على ع طلبة أو · ع ٪ حصلوا على تقدير مقبول، و ب طلبة أو · ץ \% حصلوا على تقدير ناقص، و ب طلبة أو · م \% حصلوا على تقدير ضعيف. وبعد عرض الباحثة نتيجة مجموع الضابط ومجموع التجربة. أقامت الباحثة المقارنة بين نتيجة جموع الضابط والتجربة. كما قدّمتها الباحثة في الجدول التالي:

\begin{tabular}{|c|c|c|c|c|}
\hline$d^{\wedge} 2$ & $d$ & $\mathrm{Y}$ & $\mathrm{X}$ & الرقم \\
\hline VYq & rV & Vr & $9 \wedge$ & 1 \\
\hline vrq & TV & 79 & 97 & r \\
\hline vrq & rV & $7 \varepsilon$ & 91 & r \\
\hline$\vee \wedge \varepsilon$ & rᄉ & 71 & 19 & $\varepsilon$ \\
\hline $1 \cdot 19$ & rr & 00 & $\Lambda \wedge$ & 0 \\
\hline 971 & r & or & $\Lambda \varepsilon$ & 7 \\
\hline 1107 & $r \varepsilon$ & 0. & $\Lambda \varepsilon$ & V \\
\hline $17 \ldots$ & $\varepsilon$. & $\varepsilon$ & N & $\Lambda$ \\
\hline $17 \ldots$ & $\varepsilon$. & $\varepsilon$. & $\Lambda$. & 9 \\
\hline $1 \cdot r \varepsilon$ & rr & $\varepsilon$. & VT & 1. \\
\hline $1 \cdot \varepsilon \cdot 1$ & r 19 & $0 \leqslant 7$ & 170 & المجموع \\
\hline $1 \cdot\{\cdot 61$ & 1169 & $0 \leqslant 67$ & 17.0 & المتوسط \\
\hline
\end{tabular}

الجدول r : مقارنة نتيجة مجموع الضابط والتجربة من الموضوع الثاني

$$
\begin{aligned}
& \text { البيانات: } \\
& \text { X } \\
& \text { Y } \\
& \text { D التفاوت بين نتيجة جموع الضابط وبجموع التجربة }
\end{aligned}
$$


بناء على العرض السابق وجدت الباحثة التفاوت بين نتيجة مجموع الضابط ونتيجة مجموع

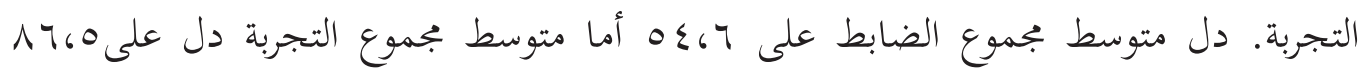
وحصلت الباحثة الثفاوت بينها على 9، آب. ولتحليل تلك النتائج استخدمت الباحثة معادلة التاء

$$
\begin{gathered}
t=\frac{M d}{\sqrt{\frac{\sum x^{2} d}{N(N-1)}}} \\
t=\frac{54: 6}{\sqrt{\frac{1040,1}{10(10-1)}}} \\
t=\frac{54: 6}{\sqrt{\frac{1040,1}{10(9)}}} \\
t=\frac{54: 6}{\sqrt{\frac{1040,1}{90}}} \\
t=\frac{54: 6}{\sqrt{11.5}} \\
16 \cdot 5=\frac{54: 6}{3: 3}
\end{gathered}
$$

كانت فرضية البحث التي قدمتها الباحثة هي أن المواد التعليمية التي استخدمتها الباحثة

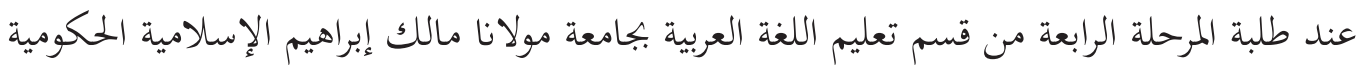

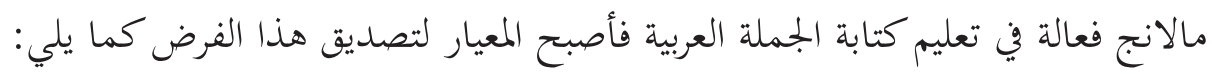
أ) إذا كانت درجة تاء الحساب أكبر من درجة تاء الجدول (t-table) فالفرض مقبول، أي أن المواد التعليمية المستخدمة فعالة في تعليم كتابة الجملة العربية.

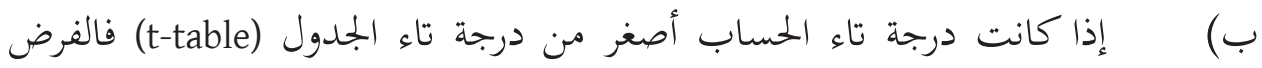
مرفوض، أي أن المواد التعليمية المستخدمة فعالة في تعليم كتابة الجملة العربية. قبل أن تختبر الباحثة فرضية البحث بذلك المعيار، عرضت الباحثة البيانات الآتية:

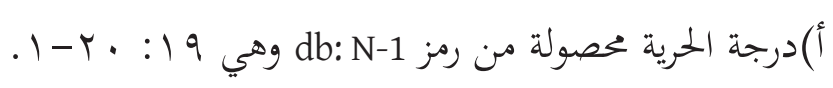




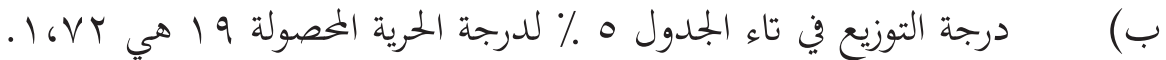
الدرحة المحصولة من تاء الحساب:

حصلت الباحثة من العرض السابق أن درجة تاء الحساب (0، 1 1 ) أكبر من تاء الجدول على مستوى التصديق ه \% ( IVYT ). بمعنى أن الفرض مقبول أي أن المواد التعليمية المستخدمة فعالة في تعليم كتابة الجملة العربية.

وقد قامت الباحثة بالتحربية الميدانية بالمواد التعليمية على الموضوعين وحصلت الباحثة أن الفرض مقبول من التجريبين السابقين أي أن المواد التعليمية فعالة في تعليم كتابة الجملة العربية. هـ - مناقشة البحث

ولمعرفة فعالية المواد التعليمية المستخدمة في تعليم كتابة الجملة الاسمية والفعلية قامت الباحثة intact group بالتجربة الميدانية. قامت الباحثة بتربة الموضوعين من المواد التعليمية بالمنهج comparison وهي انقسام الفصل إلى مجموعتين التجريبية والضابطة (Sugiono, 7 ( • r: V0). وقامت الباحثة بعملية التجرية في الفصل ب بعشرين طالبا واستخدمت الباحثة ت- الاختبار في تحليل البيانات. وحصلت الباحثة على نتيجة ت الحساب 7 T، 76 وهي أكبر من نتيجة ت الجلدول 1.VY، و Vذه النتيجة تدل على أن المواد التعليمية المستخدمة فعالة. وفي الموضوع الثاني قامت الباحثة بعملية التجربة بنفس المنهج وحصلت على نتيجة ت الحساب 7 (10 ه وهي أكبر من نتيجة ت الجدول 16 VY وهذه النتيجة تدل على أن المواد التعليمية المستخدمة فعالة. ودلت نتيجة الاختبار من الموضوعين على أن المواد التعليمية فعالة وثابتة.

وهذه النتيجة مطابقة بفروض الدراسة التقابلية على أن المواد التعليمية الجيدة هي المادة المنظمة عبر الاختلافات والمتشاهات بين اللغة السليقة واللغة المدروسة. اعتمدت الباحثة على فروض الدراسة التقابلية التي تشير إلى أن التراكيب المشابهة ستؤدي إلى سهولة الطلبة في تعليم اللغة الأجنبية وبالعكس التراكيب المختلفة ستؤدي إلى صعوبة الطلبة في تعليم اللغة الأجنبية 
(. Henry Guntur Tarigan), 999 1: ه ه). فاختارت المواد التعليمية على أساس الدراسة التقابلية

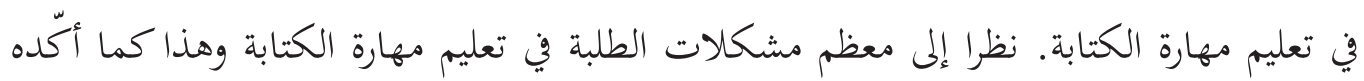

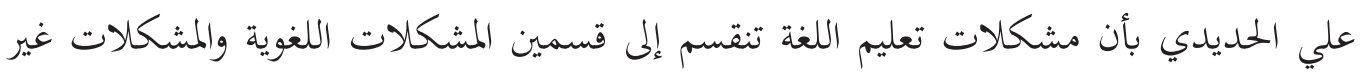

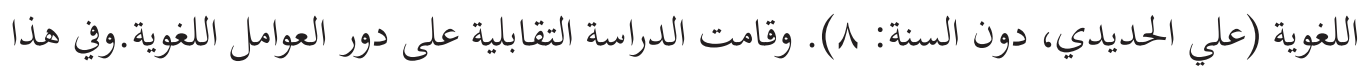

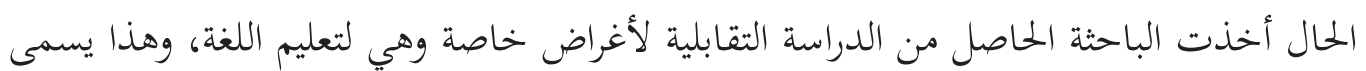

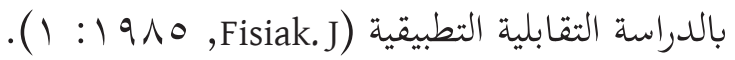
والمشكلات غير اللغوية لتعليم اللغة الأجنبية خاصة في تعليم مهارة الكتابة كثيرة وضرورة

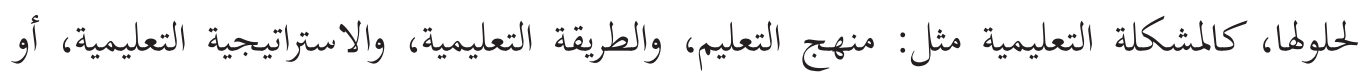
مشكلة الثقافة وما أشبه ذلك.

\section{و - خلاصة البحث}

قامت الباحثة بتجربة موضوعين من المواد التعليمية بالمنهج intact group comparison

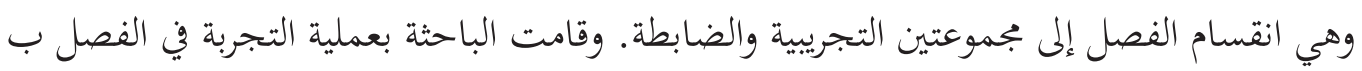

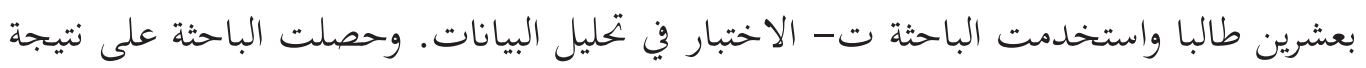

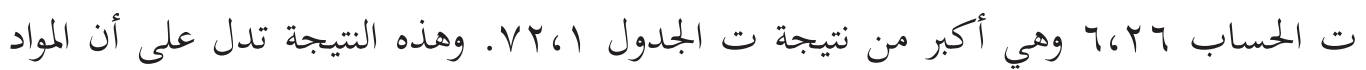
التعليمية المستخدمة فعالة. وفي الموضوع الثاني قامت الباحثة بعملية التجربة بنفس المنهج وحصلت

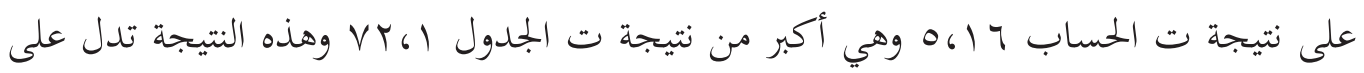
أن المواد التعليمية المستخدمة فعالة. ودلت نتيجة الاختبار من الموضوعين على أن المواد التعليمية

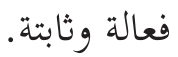




\section{قائمة المراجع}

أ-- المراجع العربية

بدر، أحم. ب ب 19 ا م. أصول البحث العلمي ومناهجه، كويت: وكالة المطبوعات عبد الله الحرمي. البشير، أحمد بن عبد الله. 19 1 1 م. التحليل التقابلي بين النظرية والتطبيق، الموجه في تعليم اللغة العربية لغير الناطقين بما ب، جاكرتا: LIPIA

الحديدي، علي. مشكلات تعليم اللغة العربية لغير العرب، القاهرة: دار الكاتب العربي. الخولي، محمد علي. r 191 م. أساليب تدريس اللغة العربية، ط. r، الرياض: المملكة العربية

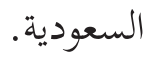

ربابعة، إبراهيم علي. مهارة الكتابة ونماذج تعليمها، الألوكة.

طعيمة، رشدي أحمد. تعليم اللغة العربية لغير لناطقين بها مناهجه وأساليبه، مصر: جامعة المنصورة. . 1919 م. تعليم العربية لغير الناطقين هما؛ مناهجه وأساليبه، رباط:

منشورات المنظمة الإسلامية للتربية والعلوم والثقافة إيسيسكو. لعريه.

الفرجاني، عبد العظيم عبد السلام. ؟ . . T م. تكنولوجيا إنتاج المواد التعليمية، القاهرة: دار غريب. الفوزان، عبد الرحمن إبراهيم. ^ץ؟ ا هـ. إعداد مواد تعليم اللغة العربية لغير الناطقين هما، رياض: ملك سعود العربي.

النحيحي، محمد لبيب ومحمد منير مرسي. r 91 ا م. البحث والتربية أصوله ومناهجه، القاهرة:

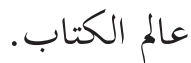
يونس، فتحي علي ومحمد عبد الرؤوف الشيخ. ب. . . ب م. المرجع، القاهرة: مكتبة وهبة.

Arikunto, Suharsimi. 1980. Prosedur Penelitian. Yogyakarta: Bina Aksara. Chaer, Abdul dan Leoni Agustina. 2010. Sosiolingustik Perkenalan Awal, Jakarta: Rineka Cipta.

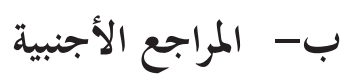


Fisiak, J. 1985. Contrastive Linguistics and The Language Teacher, Oxford: Pergaman Press.

Henry Guntur Tarigan. 1992. Pengajaran Analisis Kontrastif Bahasa, Bandung: Angkasa.

2008. Menulis; Sebagai Keterampilan Berbahasa, Bandung: Angkasa.

Parera, Jos Daniel. 1997. Linguistik Eduksional, Jakarta: Penerbit Erlangga.

Sudijono, Anas. 2007. Metodologi Penelitian Pendidikan. Jakarta: Bumi Aksara.

Sugiono. 2016. Metode Penelitian Kuantitatif Kualitatif dan R \& D, Cetakan Ke-24, Bandung: Alfabeta.

Sukardi. 2007. Metodologi Penelitian Pendidikan, Jakarta: Bumi Aksara. 